\title{
Acknowledgement to Reviewers of Fire in 2019
}

Fire Editorial Office

MDPI, St. Alban-Anlage 66, 4052 Basel, Switzerland

Published: 21 January 2020

The editorial team greatly appreciates the reviewers who have dedicated their considerable time and expertise to the journal's rigorous editorial process over the past 12 months, regardless of whether the papers are finally published or not. In 2019, a total of 58 papers were published in the journal, with a median time to first decision of 14 days and a median time from submission to publication of 37.5 days. The editors would like to express their sincere gratitude to the following reviewers for their generous contribution in 2019:

Abelsson, Anna

Aleksandrov, Mitko

Alves, Almeida Danilo Roberti

Ambrosia, Vincent G.

Anderson, Lysanna

Ascoli, Davide

Asumadu-Sarkodie, Samuel

Badik, Kevin J.

Bajocco, Sofia

Baker, Kirk

Benali, Akli

Berryman, Erin

Beverly, Jen

Boer, Matthias

Boisrame, Gabrielle

Brenkert-Smith, Hannah

Bright, Benjamin C.

Brown, Peter

Brugger, Sandra

Bui, Dieu Tien

Bush, Mark

Castro Santana, Níckolas

Chang, $\mathrm{Yu}$

Charney, Jay

Cho, Jaeil

Chow, Wan-ki

Christianson, Amy

Cirulis, Brett

Copes-Gerbitz, Kelsey

Coughlan, Michael R.

Crowley, Morgan A.

Cruz, Miguel

Cunillera-Montcusí, David

Czymzik, Markus

Dimyadi, Johannes

Dombrovsky, Leonid
Dwomoh, Francis

Eburn, Michael

Fernandes, Paulo

Fishler, Hillary

Floyd, Jason E.

Forkel, Matthias

Fornacca, Davide

Fusco, Emily J.

Garcia-Lazaro, Jose Rafael

Gauthier, Sylvie

Gibson, Rebecca

Glauberman, Gary

Gollner, Michael

Goodrick, Scott L.

Gould, James S.

Granath, Gustaf

Hadden, Rory

Hamilton, Matthew

Hankins, Don

Hassan, Quazi K.

Hassanain, Mohammad

Häyrén, Anneli

Heinsch, Faith Ann

Henderson, Sarah

Hoff, Valentijn

Hollis, Jennifer

Huang, Zhongwei

Imperatore, Pasquale

Inderpreet Kaur, Inderpreet

Iniguez, Jose M.

Iriarte-Goñi, Iñaki

Jahn, Jody L.S.

Jia, Shenyue

Johnston, James D.

Johnston, Lynn

Kaplan, Jed O. 
Kasymov, Denis

Kochtubajda, Bob

Kolden, Crystal

Kovács, Ferenc

Kramer, H. Anu

Król, Małgorzata

Lai, Chun-Ta

Lake, Frank K.

Lamelas, María Teresa

Lawhon, Lydia A.

Levine, Carrie

Leys, Bérangère

Li, Kaiyuan

Liang, Shuang

Liu, Tianjia

López Saéz, José Antonio

Loudermilk, Louise

Lowman, Lauren

Ma, Qin

Maleta, Yulia

Manzano, Saúl

Mariani, Michela

Marsh, Suzanne M.

Martin-StPaul, Nicolas K.

Matthews, Stuart

May, Andrew

McDaniel, Virginia

Meldrum, James

Metallinou, Maria-Monika

Ming, Jing

Mockrin, Miranda

Molina, Juan Ramón

Molinari, Chiara

Moran, Christopher J.

Morcos, Maged

Mouillot, Florent

Moya, Daniel

Murray, Brad

Navarro, Gabriel

Nguyen, Mai H.

North, Malcolm

Nunes, A. N.

Nunes, Silvia

O'Connor, Christopher

Palaiologou, Palaiologos

Parida, Bikash Rajan

Parker, Richard J.

Pellatt, Marlow

Penney, Greg

Peterson, Seth H.
Piemonte, Andrea

Qi, Yi

Quinn, Casey

Rafinazari, Amir

Rahman, Shahriar

Ramani, Alwar

Raposo, Jorge Rafael Nogueira

Raptis, Dimitrios

Rodman, Kyle

Rogeau, Marie-Pierre

Rossa, Carlos G.

Rossi, Jean-Louis

Ruecker, Gernot

Ruffault, Julien

Saito, Yoko

Saladyga, Thomas

Sazib, Nazmus

Sharples, Jason

Silva Junior, Celso Henrique Leite

Smith, Jordan

Sopko, Paul A.

Speer, Jim

Srock, Alan F.

Stefanidis, Kostas Stefanidis

Strahan, Ken

Syphard, Alexandra D

Talhelm, Alan F.

Talucci, Anna

Tanase, Mihai

Tedim, Fantina

Thomas-Van Gundy, Melissa

Timberlake, Thomas J.

Trucchia, Andrea

Tucker, Madelyn

Valero, Mario

Van Etten, Eddie

Varner, Morgan

Vega-Nieva, Daniel

Verkaik, Iraima

Vicente, Sandoval

Viedma, Olga

Węgrzyński, Wojciech

Wollstein, Katherine

$\mathrm{Wu}$, Zhuoting

Young, Adam

Yuen, Anthony Chun Yin

Yurkonis, Kathryn

Zavaleta, Pascal

Zhou, Qiang

Zhou, Xiaochi (Joe) 
(C) 2020 by the author. Licensee MDPI, Basel, Switzerland. This article is an open access article distributed under the terms and conditions of the Creative Commons Attribution (CC BY) license (http://creativecommons.org/licenses/by/4.0/). 